\title{
CHOLEDOCHAL CYST: A CASE REPORT AND DISCUSSION OF AETIOLOGY
}

\author{
BY \\ R. H. BURNELL and G. B. MARKEY \\ From the Department of Child Health, University of Adelaide, Adelaide Children's Hospital, \\ North Adelaide, South Australia
}

(RECEIVED FOR PUBLICATION OCTOBER 20, 1964)

Choledochal cyst is an uncommon condition, though more than 400 cases have been described, the first by Vater in 1723 (Aird, 1957). In recent years, as the nature of the disease has become more widely recognized, the frequency of reports has increased considerably. Parallel with this, and with each succeeding review, the number of theories of aetiology has multiplied.

As Gross (1953) states, 'With such a multiplicity of explanations it it entirely possible that we are dealing with a heterogeneous group of lesions which may be produced in several ways, for while many of the theories are plausible, no one explanation fulfils the condition found in all cases.'

A recent case in this hospital, diagnosed preoperatively as suffering from choledochal cyst, and coincidentally possessing a congenital hydroureter and hydronephrosis, has suggested another cause. Indeed, a review of some of the published reports suggests that this hypothesis may be a plausible explanation for quite a large number of cases.

\section{Case Report}

The patient, a male aged 1 year and 4 months, was admitted to the Adelaide Children's Hospital on January 6,1964 . He was the first and only child of Australian parents, the product of a forceps-assisted birth. His birth weight was $4 \mathrm{~kg}$. and he had progressed normally, with no significant illness, until two days before admission.

At this time he developed intermittent abdominal pain, vomiting, and constipation. Examination on admission showed a well-nourished, well-hydrated baby. No significant cause for his illness could be found. Abdominal findings were entirely negative. Microscopic examination of the urine, and the blood picture, were normal.

Intermittent abdominal colic and vomiting continued without detectable cause until January 9, when jaundice was first noticed. Serum bilirubin at this time was $2 \cdot 4$ $\mathrm{mg} . / 100 \mathrm{ml}$.

Barium meal performed on January 9, showed marked displacement of the duodenum anteriorly and medially, with no evidence of obstruction.
At intravenous cholangiogram on January 10, the liver excreted dye in fair concentration, outlining dilated hepatic ducts, and, apparently, a small choledochal cyst in the region of the common bile-duct. The gall-bladder appeared normal. A coincidental finding was gross left hydronephrosis and hydroureter apparently due to uretero-vesical obstruction. The right kidney and ureter appeared normal.

It was thought that, of the two lesions found, the renal tract obstruction was the more urgent. Cystoscopy on January 13 confirmed that the obstruction was at the lower end of the left ureter, the right ureteric orifice and bladder being normal. After this examination, he developed a high fever, apparently due to urinary tract infection. The only suitable antibiotic for the responsible organism, a $B$. proteus, was chloramphenicol, of which a full course was given with satisfactory response.

On January 21 a simple transvesical left ureteric meatotomy was performed, accepting the risk of later ureteric reflux. Recovery from this operation was uneventful.

However, the patient continued miserable, with intermittent abdominal pain and occasional fever. This was at first thought to be due to persisting urinary infection, though repeated examination of the urine showed a progressive return to normal. In view of the possibility of ascending cholangitis being a cause of the continuing fever, it was decided to proceed with the definitive operation on the biliary system.

The second operation was performed on February 7 , through a horizontal rectus-cutting incision. There was a moderate excess of bile-stained peritoneal fluid. The region of the common bile-duct and porta hepatis was walled off by omental adhesions, producing a cyst-like cavity containing dark green bile. The gall-bladder appeared normal, though somewhat thick-walled.

The adhesions were broken down (Fig.), revealing two ragged perforations in the anterior wall of the common bile-duct, at and just distal to its junction with the cystic duct. The perforations were of approximately equal size and measured 3-4 mm. in diameter. The more proximal perforation seemed to involve the distal part of the cystic duct. The remainder of the common bile-duct seemed only slightly dilated, but the anterior duct wall surrounding the perforations and extending both proximally and 


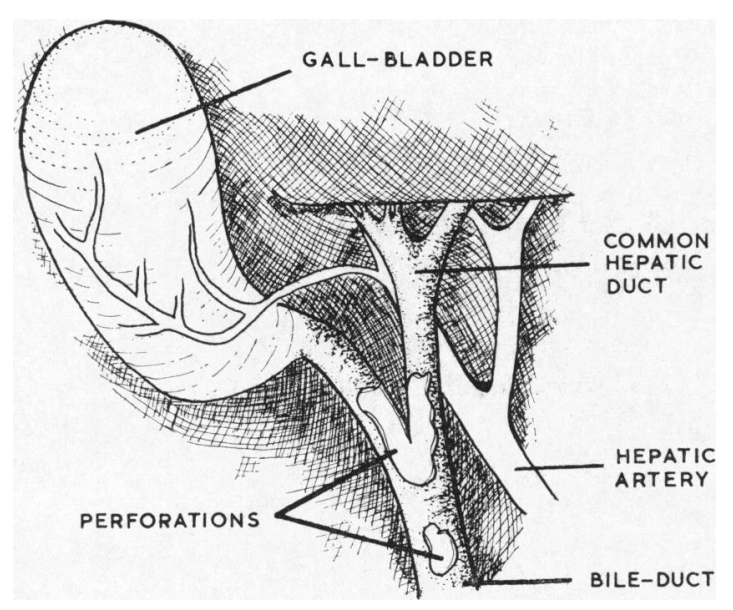

Fig.-Diagrammatic representation of conditions found at operation.

distally about $1 \mathrm{~cm}$. appeared almost necrotic, rather suggestive of an infarct.

Exploration of the bile-ducts and choledochogram failed to prove patency into the duodenum, due to obstruction of unknown nature just proximal to the sphincter. The perforations were joined by excising part of the anterior wall of the common bile-duct between them, and choledocho-jejunostomy was performed on the Roux-Y principle. No other disease could be found so the wound was closed with drainage to the operation site.

Post-operative recovery was quite uneventful, and he was discharged home on February 20, progressing well. When last seen on May 4, he continued well, and was gaining weight satisfactorily. Serum bilirubin at this time was $0.8 \mathrm{mg} . / 100 \mathrm{ml}$.

\section{Discussion}

Spontaneous perforation of the bile-ducts, like choledochal cyst, is rare. Johnston (1961) in a review of neonatal cases found only 12 . However, it seemed likely, as suggested by Aird (1957) and others, that many cases of spontaneous bile peritonitis are due to small perforations in the bile-duct system which either seal off before discovery, or are missed at operation.

A possible relation between spontaneous perforation of the bile-ducts and choledochal cyst has been suggested previously by Johnston (1961) and Caulfield (1936), and the present case, as well as those reported, provide some evidence in support of this.

Both conditions are commoner in females. Aird (1957) states that choledochal cyst is four times more common in females, and this ratio is supported by most other writers. An analysis of cases collected by McLaughlin (1946) shows that perforation of the bile-ducts and idiopathic bile peritonitis in the absence of other gross biliary tract disease is also four times more common in females. In the series of McWilliams (1912), including many cases with stones, there are twice as many females as males.

Allied to this evidence, both diseases are associated with the younger age-groups. Thus Alonso-Lej, Rever, and Pessagno (1959) found that $82 \%$ of cases of choledochal cyst were diagnosed before the age of 30 years. Johnston (1961) states that spontaneous perforation of the bile-ducts is found almost exclusively in newborn infants. Though this is not strictly true, and exact figures of age incidence of idiopathic bile peritonitis are not quoted or are not available, an analysis of the cases of McLaughlin (1946), Cope (1925), McWilliams (1912), and Johnston (1961), shows a similar age incidence. A significant fact related to age incidence is that choledochal cyst is singularly uncommon in newborn infants, surely unusual for a purely congenital malformation of this nature. Thus Alonso-Lej et al. (1959) could find only 5 cases diagnosed before the age of 3 months, from a total of 92 . In the same series $18 \%$ were found before the age of 1 year.

As Aird (1957) and McLaughlin (1946) state, sterile bile produces only mild peritonitis in humans, though its ability to stimulate fibrosis with prolonged action is well known. Because of this it is suggested that leakage of bile, in the absence of other gross pathology of the biliary tree, is, in many cases, effectively contained by the normal defences of the peritoneal cavity. Such an event would produce an illness not unlike acute cholecystitis or even acute hepatitis, and would be expected to settle without operative treatment. The histories of many reported cases of choledochal cyst do start with such an incident, and give a history extending over weeks or months, but not often years. This suggests an acquired condition, and certainly not one present since birth. Even though the basic defect may be congenital, such as the 'protodilatational state' as postulated by Alonso-Lej et al. (1959), it seems likely that the cyst is a secondary manifestation.

Although the evidence is incomplete, it seems certain that a high percentage of choledochal cysts have no, or little, epithelial lining, and a fibrous wall containing no smooth muscle (Gross, 1933, 1953; Alonso-Lej et al., 1959; Gilliland, Holloway, and Lange, 1950; Wrightson, 1953; Shallow, Eger, and Wagner, 1943; Tsardakas, and Robnett, 1956). This finding is usually explained by the occurrence of infection, but it can equally well be explained by the present theory which would be untenable if there was always a complete epithelium. In fact, many cases give a relatively short history, and produce very little clinical evidence of infection severe enough to 
produce ulceration within the cyst. Certainly epithelial loss is not a usual feature of cholangitis.

A significant percentage of cases of choledochal cyst show some intrinsic obstruction to the distal bile-duct, usually incomplete. This could also be related to bile leakage or perforation of the duct, though, as Aird (1957) states, obstruction of the common bile-duct rarely leads to such an event. The present case, however, does fit into this category, but as is so often the case, the presence of a normal gall-bladder suggests that obstruction is only a minor factor in aetiology.

Thus the present hypothesis is that many, but not all, choledochal cysts are formed following spontaneous perforation of the bile-duct. The subsequent leakage of bile is then contained by the usual defences within the peritoneal cavity, probably mainly by omental adhesions around the affected area. Cases operated on at this stage are classified as spontaneous perforation of the bile-duct or bile peritonitis. However, once the bile leakage is contained, symptoms subside and the true nature of the condition is hidden. This is particularly the case in infants, where symptoms are few, and signs of peritonitis often masked or missed. Progressive thickening of the wall surrounding the extravasation eventually produces the 'cyst' found at later operation. If operation is delayed long enough it may even be possible to find some patches of epithelium spreading from the adjacent duct.

The cyst retains its connexion with the bile-duct system via the original perforation, preventing complete absorption of the contained bile, or conversion to a true abscess.

Collapse of the cyst is prevented by two main factors. One is the narrowness of the connexion between the cyst and the bile-duct. The other is that the wall of the cyst will not contract, both because of the absence of muscle in its wall, and also because of the positive pressure within the biliary system, maintained by the gall-bladder which is usually normal in this condition.

Removal of this positive pressure is the basis of the usual method of operative treatment. As Gross (1953) states, 'There has been ample opportunity to demonstrate the potentiality of the cyst to shrink to small dimensions when adequate drainage has been established.' This is not the behaviour of a congenital cyst.

The present case provided a convincing example of the course of events. Although intravenous cholangiography before operation had shown the picture of choledochal cyst, at operation it was recognized that the condition was spontaneous perforation of the bile-duct. The 'cyst' was well on the way to completion by the time of operation, and it is suggested that a further delay of two or three weeks would have produced a classical picture of choledochal cyst.

\section{Summary}

A case of spontaneous perforation of the common bile-duct in an infant is presented.

Coincidental left hydronephrosis and hydroureter is described.

A causal relation between local bile peritonitis and choledochal cyst is suggested and discussed.

Our thanks are due to Professor G. M. Maxwell and Mr. G. G. Wyllie, under whose care the patient was admitted; and to Dr. W. McCoy for the radiological investigations.

\section{REFERENCES}

Aird, I. (1957). A Companion in Surgical Studies, 2nd ed., p. 1012. Livingstone, Edinburgh.

Alonso-Lej, F., and Rever, W. B., Jr., and Pessagno, D. J. (1959). Congenital choledochal cyst, with a report of 2 , and an analysis of 94, cases. Int. Abstr. Surg., 108, 1.

Caulfield, E. (1936). Bile peritonitis in infancy. Amer. J. Dis. Child., $52,1348$.

Cope, Z. (1925). Extravasation of bile. Brit. J. Surg., 13, 120.

Gilliland, M. B., Holloway, C. E., and Lange, J. H. (1950). Congenital choledochus cyst. J. Pediat., 37, 387.

Gross, R. E. (1933). Idiopathic dilatation of the common bile duct in children. ibid., 3, 730 .

- (1953). The Surgery of Infancy and Childhood. Saunders, Philadelphia.

Johnston, J. H. (1961). Spontaneous perforation of the common bile duct in infancy. Brit. J. Surg., 48, 532.

McLaughlin, E. F. (1946). Choledochus cyst. Ann. Surg., 123, 1047.

McWilliams, C. A. (1912). Acute, spontaneous perforation of the biliary system into the free peritoneal cavity. ibid., 55, 235.

Shallow, T. A., Eger, S. A., and Wagner, F. B. (1943). Congenital cystic dilatation of the common bile duct. ibid., 117, 355.

Tsardakas, E., and Robnett, A. H. (1956). Congenital cystic dilation of the common bile duct. Arch. Surg., 72, 311.

Wrightson, P. (1953). Congenital cystic dilatation of the common bile duct: a case occurring in infancy. Aust. N.Z.J. Surg., 23, 110. 